\title{
Measurements and Analysis of Self-similarity Based on Experimental Data
}

\author{
Hongfei $\mathrm{Liu}^{1}$, Lijun $\mathrm{Li}^{2, *}$ and Jianchao $\mathrm{Gan}^{3}$ \\ ${ }^{1}$ Chongqing Creation Vocation College, Chongqing, China \\ ${ }^{2}$ College of Mechanical Engineering Chongqing University, Chongqing, China \\ ${ }^{3}$ Chongqing Communication Institute. Chongqing, 400035, China \\ ${ }^{*}$ Corresponding author
}

\begin{abstract}
A method about the fractal characterization of nonstationary time series, which is based on measure by dividing the sequence into many segments and not sensitive to embedding dimension, is proposed in this letter. There are several differences in our method compare with above methods. To analyze several experimental data we find that the new method can effectively measure the fractal scale coefficient and detect present of the object in land clutter.
\end{abstract}

Keywords- self-similarity; scale coefficient strange attractor; land clutter

\section{INTRODUCTION}

Study of chaotic dynamics has been of interest to many physicists, communication engineering and it has received attention from researchers working in other fields of science and engineering. Besides exploring the precise nature of chaotic behavior of many dynamics systems, researchers desire to set up suitable methods to compute the dimension of strange attractors. The attractors of chaotic dynamical systems are also known to fractal properties. One of classical algorithm which computes correlation dimension [4] is Grassberger and Procaccia, the Grassberger-Procaccia Algorithm is used for estimating the correlation dimension of some fractal measure from a given set of points randomly distributed. But there are many strict limits [5-11] to the accuracy of calculation.

For whole fractal set there are many algorithms such as the detrended fluctuation analysis (DFA) [12] and wavelet transformation [13] and defining method [1] (DM) for identification of the self-similarity set. The part methods above require plenty of data for the accuracy of calculation and depend on number of embedding dimension. However, another challenge facing investigators applying this type of fractal analysis to physiologic data is that these time series are often highly non-stationary. A simplified and general definition characterizes a time series as stationary if the mean, standard deviation and higher moments, as well as the correlation functions are invariant under time translation. Signals that do not obey these conditions are nonstationary. The integration procedure will further exaggerate the non-stationarity of the original data

We develop a method about the fractal characterization of nonstationary time series, which is based on defining of selfsimilarity and method by dividing the sequence into many segments. There are several differences in our method compare with above methods. First, it is not depended upon number of embedding dimension. Secondly, computing quantity is smaller than GPA and DM owing to dividing the sequence into many segments. Thirdly, it is fit for shorter experimental data such as sunspots obtained only once. To analyze several experimental data we find that the new method can effectively measure the fractal scale coefficient and detect present of the object in land clutter.

\section{SELF-SIMILAR SETS}

A generic property of fractal sets is their asymptotic selfsimilarity at small scales. If it is supposed that a discrete signal is $\left\{s\left(t_{i}\right), t_{i} \geq 0\right\}$, then its self-similarity can be defined as follows.

Self-similarity: The signal $\left\{s\left(t_{\mathrm{i}}\right), t_{\mathrm{i}} \geq 0\right\}$ is similar at $t=t_{0}$, if and only if there exists a positive constant $H \geq 0$ and a sequence $\left\{h_{\mathrm{i}}>0, \mathrm{i}=1,2, \ldots,\right\}$ such that

$$
s\left(t_{0}+h_{i} \Delta t\right)-s\left(t_{0}\right)=h_{i}^{H}\left[s\left(t_{0}+\Delta t\right)-s\left(t_{0}\right)\right]
$$

Where, $\Delta t$ denotes sampling time interval, and $H$ is called the self-similarity scaling parameter.

If signal $\left\{\mathbf{s}\left(t_{\mathrm{i}}\right), t_{\mathrm{i}} \geq 0\right\}$ is self-similar at arbitrary $t$, then it is called self-similar signal (S.S.S). Furthermore, $1 / H$ for $\boldsymbol{S . S . S}$ is interpreted as the Hausdorff dimension $\mathrm{D}_{H}{ }^{[1]}$, namely $\mathrm{D}_{H}=1 / H$. In fact, many signals such as Hénon mapping are self-similar at only partial time $t$.

We calculate the differential of a S.S.S

$$
\begin{aligned}
& \dot{s}(t)=\lim _{\Delta t \rightarrow 0} \frac{s\left(t+h_{i} \Delta t\right)-s(t)}{h_{i} \Delta t} \\
& =\frac{1}{h_{i}^{1-H}} \lim _{\Delta t \rightarrow 0} \frac{s(t+\Delta t)-s(t)}{\Delta t}=\frac{\dot{s}(t)}{h_{i}^{1-H}} .
\end{aligned}
$$

It is evident that expression (2) holds if and only if signal $\{s(t), t \geq 0\}$ satisfies condition

$$
\dot{s}(t)=0,
$$




$$
\text { or } \dot{s}(t)= \pm \infty \text {, }
$$

For almost all $t$. The equation (3) or (4) indicates that a S.S.S must be equilibrium or divergent state almost everywhere. In fact we analyze usually the signals such as noise or random signals etc, which is divergent state and not equilibrium almost everywhere.

We can't usually observe self-similarity, namely crinkliness, in high-dimensional phase space of system. To extend one-dimensional system to m-dimensional system the delay embedding vector in the phase space is reconstructed ${ }^{[14-}$ ${ }^{15]}$ as follows.

$$
\begin{aligned}
& S\left(t_{i}\right)= \\
& {\left[\begin{array}{llll}
s\left(t_{i}\right) & s\left(t_{i}+\tau\right) & \ldots & \left.s\left(t_{i}+(m-1) \tau\right)\right], i=1,2, \ldots, M,
\end{array}\right.}
\end{aligned}
$$

Here $S \in \mathbb{R}^{\mathrm{n}}$ and $\tau$ is an appropriate time delay multiple of the sampling time interval $\Delta t$ and $\mathrm{m}$ is embedding dimension. Therefore, we can get a m-dimensional series $\left\{S\left(t_{\mathrm{i}}\right)\right.$, $\left.t_{\mathrm{i}}=1, \ldots, M\right\}$. It is proved easily that following equation holds

$$
S\left(t_{0}+h_{i} \Delta t\right)-S\left(t_{0}\right)=h_{i}{ }^{H}\left[S\left(t_{0}+\Delta t\right)-S\left(t_{0}\right)\right] .
$$

It shows that the trajectory in m-dimensional phase space is self-similar in the statistical sense: if we rescale the scales of the $\mathrm{m}$ axes of the phase space by the same factor, then the rescaled trajectory has the same statistical properties as original one.

To compute statistical self-similarity scaling parameter $H$, we divide the $M$ points series $\left\{S\left(t_{\mathrm{i}}\right), t_{\mathrm{i}}=1, \ldots, M\right\}$ into $K$ $1=\operatorname{int}(M / P)$ non-overlapping segments of equal length $P$. Since the length $M$ of the vector series in phase space is often not a multiple of the considered time scale s, a short part at the end of the vector series may remain. In order not to disregard this part, the same procedure is repeated starting from the opposite end. Therefore $K$ segments are obtained altogether. We calculate following average Euclidean norm,

$$
\begin{aligned}
F\left(h_{i}, j\right) & =<\left\|S\left(t_{0}+h_{j, i} \Delta t\right)-S\left(t_{0}\right)\right\|> \\
& =\frac{1}{P} \sum_{i=1}^{P}\left\|S\left(t_{0}+h_{j, i} \Delta t\right)-S\left(t_{0}\right)\right\|, \\
i & =1,2, \ldots P, j=1, \ldots, K,
\end{aligned}
$$

and then we calculate average over $K$ segments

$$
F\left(h_{i}\right)=\frac{1}{K} \sum_{j=1}^{K} F(i, j)
$$

Here the vertical bars denote the Euclidean norm and $<>$ indicates average over $P$ points in each segment.
If equation (6) holds, then we can take nature logarithm and get

$$
\ln \left(F\left(h_{i}\right)\right)=H \ln \left(h_{i}\right)+\ln \left(F\left(h_{1}\right)\right)
$$

The equation (9) denotes that the log-log plot is a straight line whose slope is the self-similar scale parameter $H$.

We describe the core idea of calculating self-similar scale parameter $H$. From equation (6) we expected that parameter $H$ should not change with different embedding dimension $\mathrm{m}$. In fact following simulation results proved our expectation is right.

\section{IMPORTANT RESULTS}

To calculate the self-similar scale parameter $H$ we first require reconstruct phase space by estimating delay $\tau$, which is obtained by calculating auto-information ${ }^{[16]}$. The embedding dimension is taken from 1 to 5 in following simulation.

\section{A. Self-similarity of Noise}

We will numerically treat the two cases of $1 / f^{a}$ noise that one is Gausian white noise and the other is colored noise with $\alpha=2$, and perform 100 Monte Carlo simulations, respectively. A signal with power $1 / f^{\alpha}$ spectrum has theoretic value ${ }^{[1-2]}$ $1 / H=2 /(\alpha-1)$ while $\alpha$ is subject to $1 \leq \alpha \leq 3$. Especially, the signal is white noise while $\alpha$ is subject to $\alpha=0$. The sampling points have 4096, and time delay $\tau$ is taken as 10 to noise $\alpha=2$ and 1 to white noise, and each segment has 100 points.

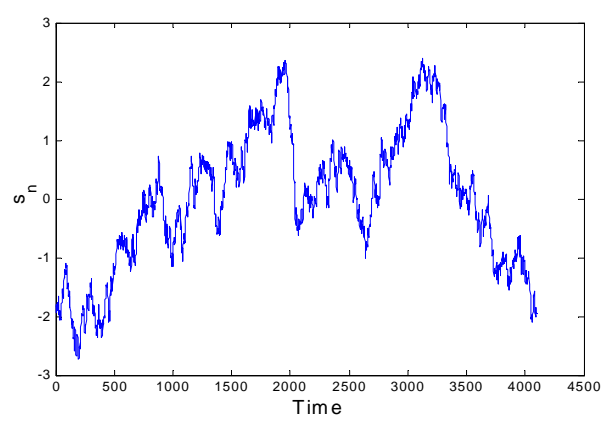

FIGURE I. THE TIME CURVE OF NOISE $\alpha=2$

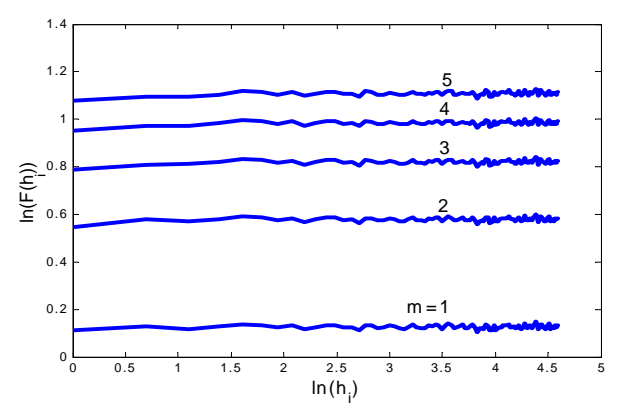

FIGURE II. THE LOG-LOG PLOT OF NOISE $\alpha=2$ 
The Fig.1 illustrates the time evolvement curve of noise $\alpha=2$, and the fig. 2 shows its log-log plot where there exists five straight lines parallel almost while embedding is from 1 to 5. The result is apparent that noise $\alpha=2$ has self-similarity and the scale parameter $\mathrm{H}$ is about 0.4569 obtained by average slope of linear fitting in 100 Monte Carlo simulations. The Table 1 concludes all data about self-similarity and indicates that Hausdorff dimension 2.1888 of noise $\alpha=2$ is close to expected dimension 2 .

The Fig 3 illustrates the time evolvement curve of white noise, and the The result is also apparent that the white noise has self-similarity and scale parameter $\mathrm{H}$ is about 0.00019 obtained by average slope of linear fitting in 100 Monte Carlo simulations. The Table 1 concludes all data about selfsimilarity and indicates that Hausdorff dimension 5528.2 of the white noise is regarded as close to expected dimension $\infty$.

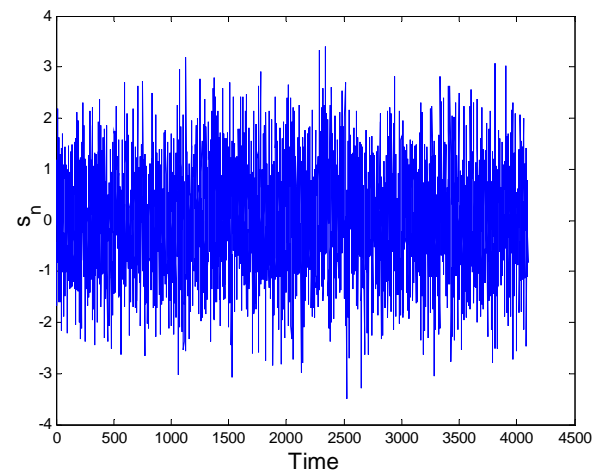

FIGURE III. THE TIME CURVE OF WHITE NOISE

The analogous results were also obtained in reference [1]. Above results show that our method is effective and noise has self-similarity.

\section{B. Self-Similarity of Period Signals}

For period sine signal $s(t)=\cos \left(\omega t+\theta_{0}\right)$, we calculate norm, e.g., absolute value in one-dimensional space

$$
\begin{aligned}
& \left|s\left(t_{0}+h_{i} \Delta t\right)-s\left(t_{0}\right)\right| \\
& =\left|\cos \left(\omega\left(t_{0}+h_{i} \Delta t\right)+\theta_{0}\right)-\cos \left(\omega t_{0}+\theta_{0}\right)\right| \\
& =\left|\cos \left(\omega t^{\prime}+\beta\right)-\cos \beta\right| \stackrel{\Delta}{=} F\left(t^{\prime}\right)
\end{aligned}
$$

Here $\omega$ is angle frequency, and $\theta_{0}$ is initial phase, and $\beta$ and $t^{\prime}$ is subject to

$$
\beta=\omega t_{0}+\theta_{0}, t^{\prime}=h_{i} \Delta t
$$

The equation (10) shows that $F\left(t^{\prime}\right)$ is a function to be the same period as original $s(t)$.
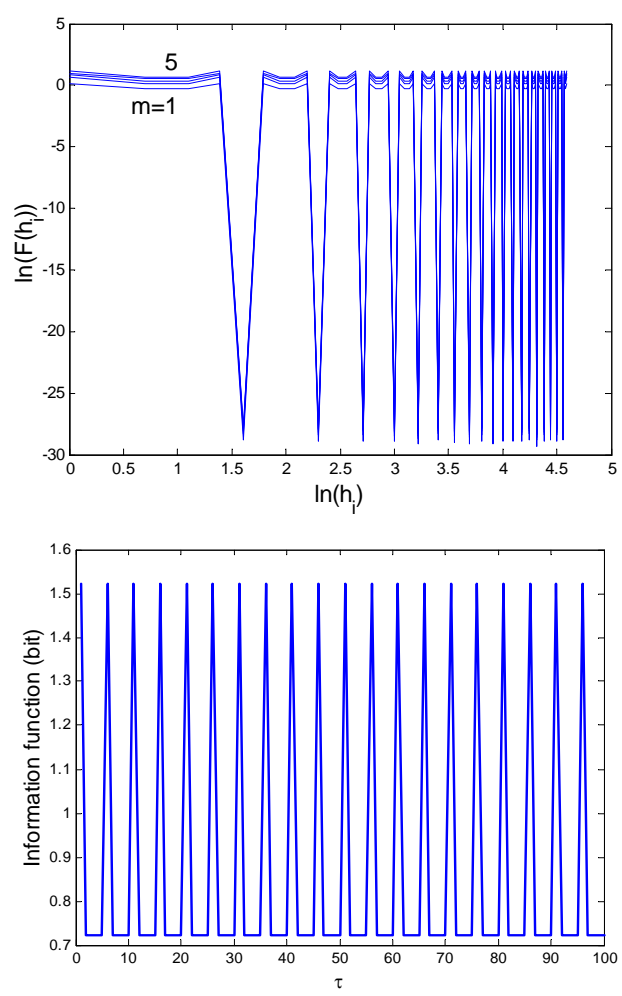

FIGURE IV. THE AUTO-INFORMATION OF SINE

We choose a sine wave whose frequency is $30 \mathrm{~Hz}$ and sampling frequency is $100 \mathrm{~Hz}$ and sampling points is 4000 and each segment has 100 points. The Fig. 4 illustrates the information function ${ }^{[16]}$ of the sine wave, and $\tau$ axis denotes delay and the vertical axis denotes information. We can get appropriate embedding delay $\tau=3$ from Fig. 3. (a) The Fig. 5illustrates the log-log plot of sine function and there is periodicity in the log-log plot. Therefore sine function has no self-similarity. In fact sine curve is smooth plot without crinkliness.

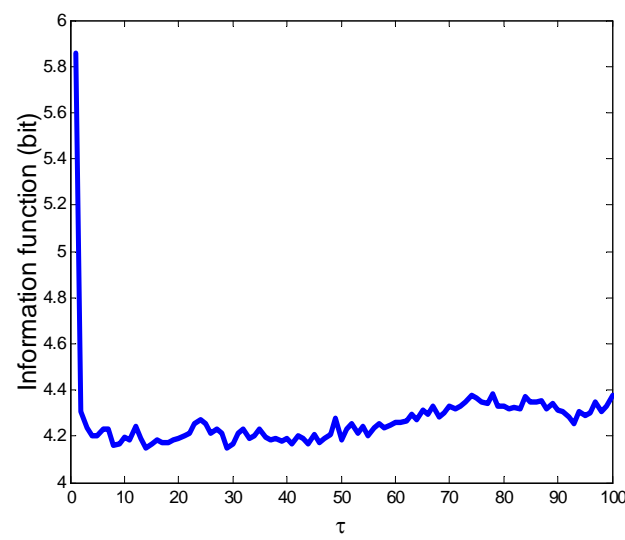

FIGURE V. THE LOG-LOG PLOT OF SINE FUNCTION 


\section{CONCLUSIONS}

In this letter, we proposed a method with small computational quantity to calculate self-similar scale parameter $\mathrm{H}$ by dividing the time series into several segments. This method is not sensitive to embedding dimension $\mathrm{m}$, but GPA is sensitive.

We numerically analyze self-similarity of noise and periodic signals, and successfully detect the echo of objects by exploiting above method. On the side, we also numerically analyze self-similarity of sunspots and laser data, and find that these data haven't self-similarity but some periodicity. At last, we detect successfully the signals from object in land clutter by exploiting our method. The applied signification of the method above is very important.

\section{ACKNOWLEDGMENTS}

This work is partly support by Chongqing Municipal Higher Education Reform Key Project (No:152079)

\section{REFERENCES}

[1] A.R.Osborne and A.Provenzale, Finite correlation dimension for stochastic sytems with power-law spectra, Physica D 35(1989) 357-381

[2] J. Theiler, Some comments on the correlation dimension of $1 / \mathrm{f} \alpha$ noise, Phys. Lett. A 155 (1991) 480-493.

[3] J. Chen, K. Y.Titus, L. Heung and J. Litva, The use of fractals for modeling EM waves scattering from rough sea surface, IEEE Transactions on Geoscience and Remote Sensing 34(1996) 966-972

[4] D Al-Shammary, I Khalil, Z Tari, AY Zomaya. Fractal selfsimilarity measurements based clustering technique for SOAP Web messages. Journal of Parallel \& Distributed Computing, 2013, 73(73):664-676.

[5] L. A. Smith, Intrinsic limits on dimension calculations, Phys. Lett. A 133 (1988) 283-288

[6] Rossi, E Sottile, P Veglia.Black-box analysis of Internet P2P applications. Peer-to-Peer Networking and Applications, 2011, 4(2):146164

[7] C. Grebogi, S.M. Hammel, J.A. Yorke and T. Sauer, Shadowing of physical trajectories in chaotic dynamics: containment and refinement, Phys. Rev. Lett. 65 (1990) 1527-1530

[8] J.Theiler, Lacunarity in a best estimator of fractal dimension. Phys. Lett. A 133 (1988) 195-200

[9] K. Judd, An improved estimator of dimension and some comments on providing confidence intervals, Physica, D 56(1992) 216-228

[10] H Lu, C Vu, X Gou. Analysis of traffic model and self-similarity for QQ in $3 G$ mobile networks.International Conference on Advanced Intelligen..., 2011:131-135

[11] R. Badii and A. Politi, Intrinsic oscillations in measuring the fractal dimension, Phys. Lett. A, 104(1984) 303-305

[12] C. K. Peng, S. V. Buldyrev, S. Havlin, M. Simons, H. E. Stanley and A. L. Goldberger, Long-range correlations in nucleotide sequence, Phys. Rev. E 49 (1994) 1685-1689

[13] H. Sam, A. T. George, L. N. Chrysostomos and J. Edmond, Self-similar identification in the time-scale domain, IEEE Trans. On Signal Processing 44(1996) 1568-1573

[14] N. H. Packard, J. P. Crutchfield, J. D. Farmer and R S. Shaw, Geometry from a time series, Phys. Rev. Lett. 45 (1980) 712-715

[15] T. Sauer, J. A. York and M. Casdagli, Embedology, Journal of Statistical Physics, 65(1992) 579-616

[16] A. M. Fraser and H. Swinney, Independent coordinates for strange attractors from mutual information, Phys. Rev. A 33(1986) 1134-1140. 